\title{
LA RESILIENCE EN TEMPS DE PANDEMIE AU \\ CAMEROUN: DE LA DIFFICILE DISTANCIATION A L'IMPOSSIBLE ENFERMEMENT SOCIAL
}

\author{
JOSEPH YANNICK MBATCHOU \\ https:/ /orcid.org/0000-0001-5396-7715 \\ Université de Yaoundé 1
}

RESUME La COVID-19 est une pandémie à l'échelle planétaire dont la propagation a été facilitée par plusieurs vecteurs qui sont à la fois naturels et humains. La dangerosité du virus, sa mutation et la difficile maîtrise de ce dernier par les hommes de sciences font du SARS-COV 2 une menace qui n'épargne aucun pays. Pour éviter l'hécatombe des sociétés comme dans les récentes pandémies qu'a connu le monde, la résilience semble être une stratégie plausible pour protéger l'humanité. Face à l'angoisse, la psychose qui alimente les populations et la déficience d'un système sanitaire, les politiques camerounais ont instauré le port de masque obligatoire dans les lieux publics non pas pour briser la résilience; mais pour s'inscrire dans la continuité de la vie. Ce projet se propose, d'étudier l'intérêt épistémique de la résilience des Camerounais face à la pandémie du coronavirus. De même, il s'agit de clarifier le concept de distanciation sociale, voire physique qui semble avoir perdu son contenu au début de la crise face aux traditions des peuples. Dans un contexte comme celui du Cameroun où le secteur informel absorbe plus de la moitié du taux de chômage, l'enfermement social a évolué en dents de scie. La réalisation de cette étude emprunte les canons de la discipline historique. Pour mener à bien ce travail, nous avons utilisé les décrets, les circulaires de la Primature et ceux du Ministére de la santé publique afin d'analyser les dispositions qui ont été prises par les hommes d'Etat pour préserver la santé des individus. Ces archives ont été confrontées aux entretiens menés auprès des acteurs économiques et des ménages dans le but, d'évaluer l'impact desdites décisions dans le quotidien des populations. Les résultats de ce travail sont pluriels et permettent de savoir, que la résilience est un concept mis sur pied au Cameroun pour protéger les populations et limiter la propagation du virus. Il ressort également que les Camerounais ont du mal à respecter la distanciation physique car cette dernière n'entre 
pas dans leurs mœurs. C'est pourquoi, l'enfermement social qui a plus ou moins réussi draine avec lui plusieurs maux socioculturels.

Mots clés: COVID-19. Pandémie. Résilience. Distanciation. Enfermement social.

\section{RESUMO A RESILIÊNCIA EM TEMPOS DE PANDEMIA NOS CAMARÕES: DO DIFÍCIL DISTANCIAMENTO AO CONFINAMENTO SOCIAL IMPOSSIVELL}

A COVID-19 é uma pandemia global cuja propagação tem sido facilitada por vários vetores que são tanto naturais quanto humanos. O perigo do vírus, sua mutação e a dificuldade de controlá-lo pelos cientistas fazem do SARS-COV 2 uma ameaça que não poupa nenhum país. A fim de evitar a hecatombe das sociedades, como nas recentes pandemias que o mundo viveu, a resiliência parece ser uma estratégia plausivel para proteger a humanidade. Diante da angústia, da psicose que alimenta as populações e da deficiência de um sistema de saúde, as políticas camaronesas introduziram o uso obrigatório de máscaras em locais públicos, não para quebrar a resiliência, mas para ser parte da continuidade da vida. Este projeto se propõe a estudar o interesse epistêmico da resiliência dos camaroneses diante da pandemia do coronavírus. Da mesma forma, trata-se de esclarecer o conceito de distanciamento social, mesmo físico, que parece ter perdido seu conteúdo no início da crise, diante das tradições do povo. Em um contexto como o de Camarões, onde o setor informal absorve mais da metade da taxa de desemprego, o confinamento social tem evoluído em convulsões e começos. Este estudo se baseia nos cânones da disciplina histórica. Para realizar este trabalho, utilizamos decretos, circulares do Gabinete do Primeiro Ministro e do Ministérios da Sapude Pública para analisar as medidas tomadas pelos estadistas para preservar a saúde dos indivíduos. Estes arquivos foram comparados com entrevistas realizadas com agentes econômicos e lares, a fim de avaliar o impacto destas decisões na vida diária da população. Os resultados deste trabalho são múltiplos e mostram que a resiliência é um conceito desenvolvido nos Camarões para proteger as populações e limitar a propagação do vírus. Mostra, também, que os camaroneses têm dificuldade em respeitar a distância física porque ela não faz parte de seu modo de vida. É por isso que o confinamento social, que teve mais ou menos sucesso, drena com ele vários males socioculturais.

Palavras-chave: COVID-19. Pandemia. Resiliência. Distanciamento. Confinamento social. 


\section{ABSTRACT RESILIENCE IN TIMES OF PANDEMIC IN CAMEROON:}

\section{FROM DIFFICULT DISTANCING TO IMPOSSIBLE SOCIAL CONFINEMENT}

The Covid 19 is a worldwide pandemic which spread was facilitated by several means of transmission which are both natural and human. The virus's dangerousness, its mutation and the difficult mastery of this latter by science men make of SARS-COV 2 to be a threat that spares no country. To avoid a massacre of population as in recent pandemics that the world experienced, resilience appears to be a plausible strategy to protect humanity. Facing anguish, psychosis which powers the populations and the deficiency of a health system, Cameroonian policies have instituted the wearing of face mask in public places to be mandatory not to break up resilience but to keep fighting to ensure life continuity. This project intends to study the epistemic interest of the resilience of Cameroonians facing the coronavirus pandemic. In the same manner, it's about making clear what social distancing is all about, and even physical which seems to have lost its essence at the start of the crisis facing various traditions of peoples. In a context as that of Cameroon in which the informal sector absorbs more than a half of the unemployment rate, social confinement has progressed by jerks and jumps. The carrying out of this study takes the patterns of historical discipline. To carry out this work, we used decrees, letters from Prime Minister's office and those from the Health Minister so as to analyse the decisions that were taken by State men in order to preserve the health of individuals. These archives have been confronted with the interviews done to people working in the economic sector and households in order to assess the impact of the said decisions in people's life in the daily basis. The results of this work are many and enable to know that resilience is a concept built up in Cameroon to protect the populations and to also limit the spread of the virus. It equally stands out that Cameroonians have trouble respecting physical distancing because this latter is not part of their culture. That's why, social confinement that has more or less succeeded drains along it several socio-cultural ills.

Keywords: Covid 19. Pandemic. Resilience. Distancing. Social confinement. 

IMPOSIBLE CONFINAMIENTO SOCIAL

La COVID-19 es una pandemia mundial cuya propagación se ha visto facilitada por varios vectores que son tanto naturales como humanos. La peligrosidad del virus, su mutación y la dificultad de controlarlo por parte de los científicos hacen del SARS-COV 2 una amenaza que no perdona a ningún país. Para evitar la hecatombe de las sociedades, como en las recientes pandemias que ha experimentado el mundo, la resiliencia parece ser una estrategia plausible para proteger a la humanidad. Ante la angustia, la psicosis que alimenta a las poblaciones y la deficiencia de un sistema de salud, las políticas del Camerún han introducido el uso obligatorio de máscaras en los lugares públicos no para romper la resistencia, sino para formar parte de la continuidad de la vida. Este proyecto se propone estudiar el interés epistémico de la resiliencia de los cameruneses frente a la pandemia de coronavirus. Asimismo, se trata de aclarar el concepto de distanciamiento social, incluso físico, que parece haber perdido su contenido al principio de la crisis frente a las tradiciones del pueblo. En un contexto como el del Camerún, en el que el sector no estructurado absorbe más de la mitad de la tasa de desempleo, el confinamiento social ha evolucionado a trompicones. Este estudio se basa en los cánones de la disciplina histórica. Para llevar a cabo este trabajo, hemos utilizado decretos, circulares de la Oficina del Primer Ministro y las del Ministerio de Salud Pública para analizar las medidas tomadas por los estadistas para preservar la salud de los individuos. Estos archivos se compararon con las entrevistas realizadas a los agentes económicos y los hogares para evaluar el impacto de estas decisiones en la vida cotidiana de la población. Los resultados de esta labor son múltiples y muestran que la resiliencia es un concepto desarrollado en el Camerún para proteger a las poblaciones y limitar la propagación del virus. También muestra que a los cameruneses les resulta dificil respetar la distancia física porque no forma parte de su modo de vida. Por eso el confinamiento social, que ha tenido más o menos éxito, drena con él varios males socioculturales.

Palabras clave: COVID-19. Pandemia. Resiliencia. Distancia. Confinamiento social. 


\section{Introduction}

Le SARS-COV 2 encore appelé le nouveau coronavirus, est une maladie infectieuse causée par le nouveau virus d'une vaste famille. Découvert récemment en Chine, à Wuhan en décembre 2019', ce virus pathogène s'attaque à l'animal comme à l'homme. C'est autant plus des raisons, qui expliquent sa mutation et sa difficile maîtrise par les hommes de sciences. Les flux intracontinentaux ont fait de cette maladie locale une pandémie, car elle touche de nombreux pays dans le monde en général et le Cameroun en particulier. Face à un virus d'une extrême dangerosité et dont la propagation est immesurable, la résilience semble être une stratégie plausible pour protéger les Camerounais. Le concept de résilience quant à lui peut se définir comme étant la capacité d'une personne à faire face aux épreuves de la vie. Pour l'Organisation Mondial de la Santé (OMS), c'est l'ensemble des initiatives développées par un patient avec l'aide d'un assistant pour protéger sa santéz. Par contre la distanciation physique est l'éloignement corporel d'un individu à un autre. Ce dernier se rapproche de l'enfermement social qui est un synonyme du confinement, mais à la différence que celui-ci n'est pas restrictif. En dehors de cette résilience qui varie d'une personne à une autre, les pouvoirs publics camerounais ont mis sur pied d'autres paradigmes permettant d'endiguer la pandémie. Ainsi, l'utilisation du socioconstructivisme permet de comprendre que face à certaines situations, voire aux pandémies, les individus développent des compétences qui leur permettent de résister à ces dernières. Cette résilience leur permet non pas seulement de riposter à la menace, mais de continuer à vaguer à leur préoccupation. De ce fait, quelle

1 "Maladie à coronavirus 2019 (COVID 19)", www.who.int/ $\mathrm{fr} /$ emergencies/diseases, 27/03/2020.

2 "La santé mentale et la résilience psychologique pendant la pandémie du COVID 19", www.who.int/fr/healttopic, 27/03/2020. est l'importance de la résilience dans la lutte contre le coronavirus au Cameroun? Pour répondre à cette préoccupation, cette étude se propose, de montrer que l'application de la distanciation sociale, voire physique a perdu son contenu au début de la crise face aux traditions des peuples locaux et que l'enfermement social en contexte camerounais a évolué en dents de scie. Dans le souci de mener à bien ce travail de recherche, nous nous attarderons sur la pandémie du coronavirus et la résilience des peuples locaux; mais aussi, sur les insuffisances de la distanciation physique et l'impossible enfermement social.

\section{La pandémie du coronavirus et la résilience des peuples locaux}

La propagation du coronavirus qui atteint le Cameroun au premier trimestre, oblige les populations à développer la résilience, qui est une arme psychothérapeutique permettant d'endiguer l'évolution du virus sur l'étendue du territoire. Cette résistance qui varie d'un individu à un autre a été amplifiée par plusieurs facteurs européocentristes et locaux.

\section{Les facteurs ayant influencés une résilience globale chez les camerounais}

Pendant plusieurs siècles, l'humanité a fait face à des crises sanitaires aux conséquences sans précédent. Il s'agit entre autre de la grippe espagnole, d'Ebola, du VIH/SIDA ou encore de la Chikungunya (BIOS, 2020). Chacune d'elle avait un caractère particulier, une propagation aigue et est parvenu autant que le coronavirus à décimer des milliers de personnes à travers le monde. Ce n'est donc pas un fait nouveau pour l'humanité qui vit aujourd'hui une crise épidé- 
mique sans égale. A la différence des crises qui ont été citées, la COVID-19 a réussi à paralyser le fonctionnement du système monde. D'autant plus que les chercheurs sont divisés et que les dirigeants des Etats et ceux de l'Union Européenne (UE) ne sont pas unanimes sur la gravité de la menace (LLYOD, 2020). Ce tâtonnement a fragilisé les Etats et a rendu vulnérable les populations des pays puissants vers celles en voies de développement.

Face à l'incapacité de maîtriser un virus mutant et de mettre fin aux lamentations que ce dernier entraine dans les pays européens, plusieurs européocentristes, acteurs économiques et organisation telle que l'Organisation Mondiale de la Santé (OMS) sont montés au créneau pour professer une sorte de fin du monde pour les Africains 3 . Pour la France, la COVID-19 pourrait être "la crise de trop, qui déstabilise durablement, voire qui met en bas des régimes fragiles (Sahel) ou en bout de course (Afrique Centrale)" (CABRIOL, 2020, s/p). A tort ou à raison, ce point de vue renferme plusieurs interrogations notamment la faiblesse scientifique du continent africain. C'est pourquoi, il y a lieu de dire que "la pandémie a donné l'occasion d'équiper les laboratoires, réduisant la dépendance à l'institut pasteur"4.

S'il est vrai que la pandémie a atteint le continent en général et le Cameroun en particulier, il faut souligner que plusieurs facteurs ont été à l'origine de la résilience chez les Camerounais. Il s'agit entre autres des idées véhiculées par les occidentaux sur l'hécatombe des Africains. Parmi ceux-ci, l'OMS, Organisation sensée protéger la vie des peuples du monde. Sa position sur la gestion de la crise a été longuement critiquée par de nombreux acteurs nationaux ${ }^{5}$ et jusqu'aujourd'hui, cette

3 Il s'agit de l'Hécatombe annoncée par l'OMS. Lire par exemple, A-G. Khalfallah, COVID 19: L'Afrique l'impossible pronostic", www.atalayar.com/fr/blog, 19/07/2020.

4 "La COVID-19 va renforcer l'autonomie scientifique de l'Afrique", www.scidev.net, 04/05/2020.

5 Le naturopathe camerounais, Linus Fotsing critiquait dernier n'a pas apporté des résultats probants sur la pandémie notamment la mise sur pied d'un vaccin pour lutter efficacement contre le virus. D'autant plus que la découverte d'un vaccin, nécessite une longue étude clinique (MBATCHOU, 2020) contrairement à ce que pensent les laboratoires occidentaux comme AstraZenesca $^{6}$. A cela s'ajoute le système de santé camerounais qui est poreux et comme tous autres systèmes africains après 60 ans d'indépendance, continu de compter sur le laboratoire pasteur en terme de logistique et d'étude sur les nouvelles crises sanitaires. Ce sont toutes ces idées, qui ont amené les Camerounais à développer la résilience pour endiguer la propagation du virus.

En réalité, la résilience n'est pas un concept nouveau dans la gestion des crises. Celui-ci a évolué dans le temps et dans l'espace pour avoir un caractère particulier dans la gestion des épidémies. En réalité, la résilience se construit en huit étapes à savoir: "la défense-protection; l'équilibre face aux tensions; l'engagement-défi; la relance; l'évaluation; la signification-évaluation; la positivité de soi et la création"?. Dans le domaine de la santé, lorsqu'un peuple est victime d'une épidémie, il développe une résistance lui permettant de faire face à la menace. C'est le cas avec la COVID-19 au Cameroun où la résilience des populations a été renforcée par les décrets des hommes d'Etats ${ }^{8}$. Malgré la crise sanitaire et

l'OMS en sens qu'en médecine traditionnelle, il n'y a pas d'étude clinique. Cf. G. Ibrahim Tounkara, "Les critiques de l'OMS passent mal en Afrique", https://m. dw.com/fr/ 18/05/2020.

6 C'est un laboratoire anglais qui pense avoir trouvé un vaccin contre le coronavirus. Ce dernier sera disponible à l'automne et conférera une protection d'un an. Cf. "Le vaccin d'AstraZeneca pourrait protéger contre le Covid-19 pendant un an", www.challenges.fr/entreprise, 16/06/2020.

7 "Résilience: définition et application aux domaines de la RSE et du développement durable", https://youmatter.world/fr/definition/resilience.

8 A compter du 18 mars 2020 et jusqu'à nouvel ordre, le président de la république du Cameroun instruit 13 mesures visant à riposter contre la pandémie du 
les mesures prises (fermeture des frontières, des débits de boisson, des sphères éducatives, port du masque et lavage des mains), les pouvoirs publics n'ont jamais confiné la population camerounaise comme cela a été fait dans les pays européens. A cet effet, un informateur anonyme souligne que le gouvernement camerounais a fait preuve de maestria dans la gestion de la crise car en évitant de confiner les populations, il a épargné le pays des conséquences socioéconomiques sans égal. Il était donc question pour les Camerounais de développer une riposte face aux effets pervers du coronavirus.

Pour soutenir cette riposte et permettre aux camerounais de résister efficacement à la crise sanitaire, les pouvoirs publics ont mis sur pied une stratégie qui a été dévoilée le 17 juin 2020 et qui s'inscrit sur une période de trois ans". Cette stratégie qui est évaluée “à 479 milliards de FCFA, repose sur 05 piliers majeurs, à savoirs la riposte sanitaire, la résilience économique et financière, l'approvisionnement stratégique, le renforcement de la recherche et de l'innovation, ainsi que la résilience sociale"10. Ce soutien du gouvernement camerounais, vise à réparer les insanités causées par le SARS-COV 2 et permettre au Cameroun en 2023 de retrouver son chemin de l'émergence. C'est pourquoi la stratégie se veut une riposte globale sur tous les secteurs de développement dont la santé qui est prioritaire afin de faire face aux prochaines crises. Cette dernière est liée à la recherche fondamentale qui doit être amplifiée en intégrant l'ordre traditionnel de la santé qui a porté du répondant dans la

Coronavirus. Cf. www.prc.cm/fr/actualités, consulté le 12 août 2020. Lire aussi, les stratégies gouvernementales de riposte face à la pandémie de coronavirus (COVID-19), www.spm.gov.cm

9 "COVID-19: Le gouvernement camerounais se dote d'une stratégie de riposte et de résilience socio-économique", 23/06/2020.

10 "COVID-19: Le gouvernement camerounais se dote d'une stratégie de riposte et de résilience socio-économique", 23/06/2020. lutte contre ladite pandémie (BELOKO, 2020)11. Il faut par-dessus tout, relancer le secteur économique et les structures sociales paralysés par la crise depuis 6 mois. Ce qui permettra un retour sur les bancs pour les tous petits dès octobre 2020 et ramener le sourire d'antan chez les Camerounais. S'il est vrai que la résilience globale est une riposte au COVID-19, il faut souligner que la démocratie sanitaire ou la culture personnelle est un facteur psychologique endiguant la propagation du nouveau coronavirus.

\section{La culture personnelle: un facteur psychologique permettant de résister à la pandémie du \\ nouveau coronavirus}

A côté de la riposte globale menée par l'Etat (création des centres d'isolement, de traitement et la ligne d'assistance locale: 1510), il y a aussi un effort personnel, voire individuel qui contribue à la lutte contre la propagation du coronavirus. Le Cameroun est un pays d'Afrique Centrale qui compte à nos jours plus 25 millions d'habitants et qui selon certains experts est l'épicentre de la pandémie en Afrique Centrale (SAVANA, 2020). Aujourd'hui le pays présente 18042 cas confirmés, 15320 guérisons et 395 décès ${ }^{12}$. Malgré cette position qui ternie l'image du Cameroun auprès de ces partenaires étrangers, il faut souligner que le pays a également le nombre le plus élevé des cas guéris ${ }^{13}$. Cet exploit peut se justifier à travers trois hypothèses notamment la chaleur qui est omniprésente (pays situé dans la zone climatique chaude). Seulement, les effets de cette

11 L'archevêque métropolitain Samuel Kléda, avait souligné au journal télévisé de la CRTV avoir guéri plus de 12000 cas de contaminés sans décès. Lire aussi, $\mathrm{F}$. Beloko, "Coronavirus: le Cameroun s'intéresse à la solution de Monseigneur Kléda", www.allodocteur.africa/ $\mathrm{cm}, 28 / 06 / 2020$.

12 "Alerte COVID-19", www.google.com

13 Ibid. 
dernière sur le virus restent une idée qui divise le monde scientifique. L'on souligne également les bienfaits de la médecine traditionnelle dans la lutte contre le coronavirus. La preuve est que tous les pays ayant un taux élevé de guérisons ou qui ont eu très peu de cas confirmés, ont mis sur pied des décoctions visant à faire face à la menace ${ }^{14}$. En temps de crise sanitaire, la culture personnelle est capitale pour résister à la pandémie.

En réalité, l'on peut avoir un exécutif proactif qui gère aussi bien une pandémie que les besoins de ses populations. Un système sanitaire bien structuré, qui met en avant la recherche fondamentale pour faire face aux crises futures. Tout ceci ne suffit pas pour riposter à une crise sanitaire sans égal comme la COVID-19. C'est le cas par exemple avec les anciens, qui ont trouvé massivement la mort dans les EHPAD en Europe et dans les CHSLD en Amérique du Nord ${ }^{15}$. Si certains paramètres socio-sanitaires peuvent justifier ces morts comme le souligne Linda Jurick ${ }^{16}$, il faut souligner que la résilience que devait développer ces patients a été fragilisée. D'autant plus que la maladie s'est abattue comme une poudrière dans ces régions du monde et ayant de nombreux antécédents médicaux, les anciens avec leur assistance n'ont pas armé une compétence physique pour résister à la pandémie. Ce point de vue peut être une controverse, seulement la préparation psychologique d'un patient lui permet de mieux gérer sa maladie.

Par contre au Cameroun, la maladie n'est présente qu'à partir de mars 202017. Ce qui donne individuellement aux Camerounais le

14 Il s'agit par exemple de Madagascar et du Cameroun qui ont su utilisés les vertus de la médecine traditionnelle pour riposter à la pandémie du coronavirus.

15 Ce sont des camps de retraites pour personnes âgées dépendantes. En réalité, cette notion est variable d'une sphère géographique à une autre.

16 Jurick, entretien du 21 avril 2020 au Québec.

17 Lire par exemple, les stratégies gouvernementales de riposte face à la pandémie de coronavirus (COVID-19), www.spm.gov.cm temps de construire une riposte physique solide. Pour faire face à cette crise sanitaire cauchemardesque, les peuples locaux développent moult résistances. C'est le cas par exemple dans les villes de Yaoundé, Douala et Bafoussam qui sont des épicentres de la pandémie et/ou aucune activité n'a été stoppée. Ceci se justifie par le fait que plus de la moitié de la population se nourrit au quotidien. Au vue de cette insuffisance, de nombreux acteurs furent contraints d'accepter de vivre avec la pandémie dans le but de pérenniser les communautés. Ce point de vue est partagé par Emmanuel Belek, qui souligne "qu'en restant confiné chez moi, l'Etat va-t-il répondre à mes besoins élémentaires?"18. Pour Odile Ngouana, "en tant que bayam sellam, je me protège toutes les fois que je suis à l'extérieur et précisément dans mon comptoir au marché huitième"19. A Ebolowa, Stéphane Mbane Bikolo déclare "qu'ici le coronavirus n'est pas une menace, c'est une affaire des grandes villes camerounaises"20. Chez les Baka communément appelés Pygmées, la riposte contre le coronavirus a été endogène c'est-à-dire propre à leur culture. Il s'agit des “mesures barrières venant de leur tradition à savoir les herbes, les feuilles et autres" (NTAP, 2020, s/p).

Toutes les informations recueillies concourent à l'idée selon laquelle, les Camerounais ont renforcé leur vécu psychosocial par rapport à la perception qu'ils ont de la pandémie. Bien que sentant leurs valeurs traditionnelles menacées, les populations ont su développer un lien étroit entre le physique et le psychique afin répondre à la menace environnementale. Pour les "non contaminés" faute de tests, voire les illuminés, il était judicieux d'adopter les comportements prodigués par les pouvoirs publics. C'est pourquoi au début de la crise sanitaire, il est facile d'avoir deux types

\footnotetext{
18 Belek, entretien du 23 mars 2020 à Yaoundé.

19 Ngouana, entretien 15 juillet 2020 à Yaoundé.

20 Mbane Bikolo, entretien 4 juin 2020 à Ebolowa.
} 
de Camerounais: ceux qui croient à l'existence de la maladie et ceux qui pensent que c'est une maladie uniquement pour les Européens. Dans ce biardisme de fait, chaque groupe développe un comportement psychosocial lui permettant de faire face à la pandémie. C'est le cas des pygmées ou des Pahouing (Beti-Bulu-Fang), qui ont fait recourt à la médecine traditionnelle d'où la consommation quotidienne des décoctions provenant de la nature.

La peur d'être confiné dans les centres d'opérations COVID-19 et/ou d'être enrôlé après un Test Rapide Covid (TRC), de nombreuses personnes atteint du coronavirus ou non se sont fait soigné à domicile avec l'aide d'un médecin de famille ${ }^{21}$. C'est ce que nous appelons ici la résilience individuelle c'est-à-dire la capacité d'un corps à résister aux micro-organismes. Pendant la maladie, l'état psychosocial doit être renforcé à l'immunité, voire à la démorésilience (GUALDE, 2015). La démorésilience est un phénomène qui permet de lier l'aspect biologique (l'homme) à la culture (l'appréhension de gestion d'une crise sanitaire) ${ }^{22}$. En dehors des cas confinés rencontrés au lendemain de leur séjour dans les centres Covid comme celui de Mandjou (Bertoua) et de Laquintinie (Douala), la plupart des patients enquêtés à domicile ou cas potentiellement confinés, relèvent que plusieurs facteurs psychosociaux ont joué un rôle dans leur rétablissement. Il s'agit entre autres de la motivation familiale, des amis, du suivi de la thérapie et des exercices physiques pour ceux qui ont choisi un mini-confinement ou qui sont en phase post-maladie. C'est également au cours de cette période, que la démorésilience est importante car elle permet aux non confinés et confinés de se renseigner davantage sur la maladie d'où l'importance des mass-médias. Pour Angélique porte, il serait judicieux pour un patient covid de se décon-

21 Mboe, entretien 15 juillet 2020 à Yaoundé. 22 Ibid. necter des chaînes de télés car celles-ci polluent son état psychique. Le point de vue de la praticienne est intéressant en ce sens qu'aux premières lueurs de la crise sanitaire du COVID-19, plusieurs médias occidentaux n'ont fait qu'attiser la psychose chez les patients. Sur un autre angle et face à un virus peu maîtrisable, il est judicieux de rester sur le qui-vive dont connecté sur les réseaux sociaux. C'est également à travers ces sources d'informations, que les premières thérapies conventionnelle (Chloroquine) ou traditionnelle (artemésia) ont été données aux populations pour lutter contre le coronavirus. Aussi, c'est à travers la culture que l'humanité a pu gérer le confinement pendant plus de cinq mois. Contre toute attente des européocentristes, la résilience chez les Camerounais apparaît comme un fait particulier dans la gestion de la crise sanitaire, en ce sens qu'elle a su consolider état psychosocial, démorésilience et tradition. Cependant, quels sont les marqueurs qui ont rendu difficile la distanciation physique et l'enfermement social des Camerounais?

\section{De la difficile distanciation physique à l'impossible enfermement des populations}

Dans la lutte contre la COVID 19 au Cameroun, plusieurs mesures ont été prises par les pouvoirs publics pour limiter la propagation du virus. Il s'agit entre autres, de la difficile distanciation sociale, voire physique et/ou l'impossible enfermement social.

\section{L'échec de l'application d'une distanciation physique au}

\section{Cameroun}

En quelques mois seulement, ce virus est parvenu à réduire la taille de la population 
mondiale. Cependant, l'absence d'une réelle thérapie face à la menace a amené les scientifiques du monde à trouver des mesures de riposte telle que la distanciation sociale, voire physique. Ce qui a permis aux autorités, d'instaurer des restrictions et de confiner de nombreuses villes telles que Wuhan, Lisbonne, Lerida pour ne que citer celles-là. Face à une propagation presque cauchemardesque et vu le nombre de morts enterrés, voire incinérés par jour, le gouvernement camerounais prend le taureau par les cornes dans l'optique de protéger ses populations. Dans les mesures barrières du 17 mars 2020, figure en droite ligne "l'interdiction des regroupements de plus de

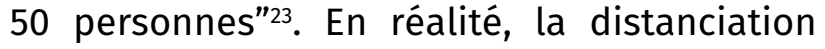
physique, distanciation sociale ou l'éloignement sanitaire est une mesure visant à éloigner les hommes les uns des autres dans les milieux publics (GOLDSTEINE et ROZEN, 2020). Cette mesure n'est pas nouvelle, car elle a toujours était utilisée par les praticiens pendant les crises épidémiques et surtout lorsque la contamination se fait par contacts physiques ou de gouttelettes ${ }^{24}$.

Aux premières lueurs de la crise, le paradigme mis en place était celui de la distanciation sociale car pour les scientifiques, il fallait confiner les populations chez elles dans l'optique d'endiguer l'évolution de la pandémie. L'on se souvient qu'en Europe, en dehors de boucler certaines villes, les autorités ont envoyé la milice s'assurer du respect de ladite restriction. Ce qui n'a pas été facile pour les populations en âge de travailler car plusieurs personnes se sont retrouvées en chômage partiel et du coup, la distanciation est devenue un fardeau. Pour les personnes contaminés et celles confinées dans les hôpitaux,

23 "Les mesures de restrictions contre le coronavirus", www.prc.cm/fr/actualités, consulté le 12 août 2020.

24 En octobre 1918, lors de la pandémie de grippe espagnole, M. C. Starkloff met en place une distanciation sociale. Cf. "Distanciation physique", www.wikipedia.org. l'éloignement social a été une fracture dans la gestion de la maladie. Au lieu d'endiguer la propagation du virus, la distanciation sociale à augmenter l'angoisse chez les personnes saines, le nombre de mort dans les pays et dont nombreux furent taxés de décès-covid. Les insuffisances de ce paradigme, voire l'isolement ont obligé les scientifiques et les autorités à revoir ledit concept et de l'adapter à l'évolution du pick de la maladie. Aujourd'hui, l'on parle désormais de distanciation physique qui est un comportement barrière, mais qui permet aux populations de briser la solitude.

Le 18 mars 2020, lorsque que les mesures de restriction prise par le président de la république entre en vigueur au Cameroun, les populations n'entendent pas laisser de côté leur tradition car pour elles la distanciation sociale, voire physique va entraîner la fracture sociale (BARROUX, 2020). C'est ainsi que certains individus, malgré les messages relayés par les pouvoirs publics et la diffusion en boucle des dangers causés par le virus dans le monde, ces derniers sont restés sceptiques et ont continué à vaguer à leurs préoccupations. C'est le cas par exemple dans les marchés et dans les débits de boisson (RABIYATOU, 2020). Un informateur anonyme souligne qu'à Obili (Yaoundé), malgré la fermeture des bars à $18 \mathrm{~h} 00$, les gens s'enfermaient dans ces lieux loin de la distanciation physique et continuaient à boire jusqu'à des heures tardives. Les résultats des enquêtes menées dans les villes de Bertoua et d'Ebolowa, affichent que plus de $70 \%$ de la population ne respectent pas les mesures barrières. Le port du masque est devenu une fantaisie à l'extérieur même pendant les cérémonies de mariage et de deuils. Le lavage des mains est facultatif uniquement pour les besoins de civilités. Malgré que le virus continu de circuler pendant cette période d'été, l'on note au Cameroun un relâ- 
chement. Aujourd'hui, c'est plus de $80 \%$ de la population qui ne respecte plus les mesures édictées par le gouvernement camerounais. L'échec de l'application de la distanciation physique, a fait que le pays soit classé en tête liste en Afrique centrale.

Au lieu de se protéger et assurer la protection des autres, la mauvaise compréhension du concept de distanciation physique à plutôt augmenter le pick de la maladie au Cameroun (BARROUX, 2020). Cet échec peut être justifié par le fait que, plus de la moitié de la population jeune exerce dans le secteur informel et que le gouvernement camerounais n'avait pas les moyens de sa politique dans la gestion de cette crise. C'est la raison pour laquelle, au lieu de réprimander comme en Europe, le gouvernement camerounais à laisser faire pour s'adosser sur le port du masque qui comme l'éloignement sanitaire n'a pas porté ces fruits. Sachant que les pouvoirs publics ne pouvaient pas répondre à tous les besoins des populations, l'enfermement social comme les autres paradigmes a évolué en dents de scie.

\section{L'enfermement social: le difficile mimétisme au Cameroun}

Le confinement ou l'enferment social est un paradigme le plus souvent associé à la résilience des individus malades ou non pendant les crises sanitaires. Ceci parce qu'en restant enfermé chez soi ou en famille, de nombreuses personnes développent une résistance parfaite contre les épidémies. Si plusieurs villes profondément touchées par le coronavirus ont été bouclées et que les déplacements ont été limités aux besoins nécessaires, les Camerounais ont dit non à ce genre de projet social qui peut être lourd de conséquences. Ainsi, le ministre de la communication Réné Sadi, souligne que "la plupart des Camerounais vivent au jour le jour. Ils n'ont pas les moyens de se ravitailler pendant 15 jours ou un mois. Nous ne pensons pas qu'il faille par mimétisme, décider de faire ce qui est fait ailleurs" (NDINGA NGOMA, 2020, s/p). Dans la même lancée, le ministre Manaouda Malachi déclare: "la chloroquine a un avantage dans le traitement du coronavirus" 25 .

Face à une crise sans égale et dont les conséquences sont à la fois politique, économique et sociale, le gouvernement camerounais qui connait ses insuffisances en matière financière refuse d'appliquer les mêmes recettes que les pays riches notamment confiner toute la population et subventionner les secteurs de développement. Les pouvoirs publics au gré des retombées, rejettent l'enfermement social. De plus que connaissant ses populations, une telle restriction n'allait pas enchanter les Camerounais. Voilà pourquoi depuis mars jusqu'à nos jours, les populations continuent de vaguer à leurs préoccupations non pas parce qu'ils ignorent l'existence de la maladie, mais parce qu'ils n'ont pas de choix. Il faille bien subvenir aux besoins des ménages et surtout les nécessités primaires des enfants aux lendemains des examens officiels.

Pour soutenir ce choix, le gouvernement propose aux Camerounais une molécule longuement utilisée et administrée aux patients depuis 1946 contre la malaria (RABIYATOU, 2020). D'après de nombreuses études faites ailleurs et ici sur l'impact de la chloroquine sur le coronavirus, il ressort que cette molécule a porté des résultats probants. Cependant, le ministre de la santé met les populations en garde contre son automédication. Motivation ou non, la chloroquine est devenue le bouclier pour les travailleurs camerounais. De plus que, dans les réseaux sociaux les praticiens sont allés jusqu'à dire que la chloroquine pouvait aussi être utilisée à titre préventif contre la

25 Ibid 
COVID-1926. Ce qui a flambé le prix de ladite molécule sur l'étendue du territoire et par ricochet accéléré sa contrefaçon et sa vente illicite d'où les sanctions affligées à certaines structures de la santé de la place (TCHUILEU, 2020). L'impossible enfermement des populations a été aussi amplifié, par l'introduction de la médecine traditionnelle dans la lutte contre le coronavirus ${ }^{27}$.

L'enferment social comme nous l'avons souligné plus haut, a évolué en dents de scie. Ceci revient à souligner que, bien qu'ayant été rejeté par le gouvernement, les migrants du retour au bercail, les intellectuels et les personnes ayant perdu un membre de leur famille dû au COVID-19 se sont confinés individuellement pour endiguer l'évolution du virus et protéger les autres. L'on pouvait alors voir dans certains quartiers, des maisons ou les portes sont restées fermés essentiellement ouvertes pour les besoins de premières nécessités. C'est également ce type de personne d'après notre observation, qui a considéré le port du masque comme une mesure barrière au coronavirus. Pour consolider ce point de vue, Loumtoné que nous avons rencontré à Emana souligne: "aujourd'hui, je rends difficilement visite à ma famille et à mes proches. Cependant, lorsque j'ai une nécessité, je mets mon masque et respecte toutes les autres mesures recommandées par l'Etat"28. Que soit à l'intérieur ou à l'extérieur, le respect de certaines mesures barrières édictées par le gouvernement est important pour endiguer la propagation du virus et sauver des vies.

En utilisant tous ces astuces de la médecine conventionnelle et traditionnelle, les Camerounais sont restés en contact permanent avec le virus; mais la résilience psychosociale

26 Ngandeu Mbouyap, entretien 15 juin 2020 à Yaoundé.

27 "Coronavirus: le Cameroun s'intéresse à la solution de Monseigneur Kléda", www.allodocteur.africa/cm, 28/06/2020.

28 Loumtoné, entretien 13 juil. 2020 à Yaoundé. associée à la démorésilience a permis aux populations de résister "efficacement" à la maladie, voire de la vaincre.

\section{Conclusion}

Au terme de ce travail qui s'intéressait à la résilience des Camerounais face à la pandémie du coronavirus, il ressort que l'application de la distanciation sociale, voire physique a perdu son contenu au début de la crise face aux traditions des peuples locaux. A cela, il faut souligner une évolution en dents de scie de l'enfermement social. Cette étude a permis de comprendre, que face à l'hécatombe projetée par l'occident sur l'Afrique en général et le Cameroun en particulier, les populations ont su développer une résistance pour endiguer le nouveau coronavirus et continuer à vaguer à leurs préoccupations. Contrairement à leurs mœurs et surtout le caractère de paupérisation qui anime le quotidien de ces peuples, la distanciation physique est l'enfermement social non pas portée des fruits comme dans les pays européens car pour ces derniers, il s'agissait de pérenniser les communautés en pleine crise sanitaire. Cette étude a une portée scientifique en sens quel permet de montrer, les astuces psychothérapeutiques développés par les Camerounais pour endiguer la crise virale, voire pour vaincre la pandémie du coronavirus. Cette préoccupation qui reste encrée dans l'esprit des occidentaux, n'est-elle pas une solution face aux maladies infectieuses comme le soulignait déjà le professeur Didier Raoult?

\section{Bibliographie}

GUALDE, Norbet. Epidémie et démorésilience. Corps, $n^{\circ} 5$, p. 15-26, 2008. Disponible sur: https://www. cairn.info/revue-corps-dilecta-2008-2-page-15.htm Consulté le: 9 juin 2020.

MBATCHOU, Joseph Yannick. La question des mé- 
dicaments illicites dans le système de santé camerounais: le cas des villes de Yaoundé, Douala et Buea (1916-2014). 2020, 420f. Thèse (Histoire), Doctorat/Ph. D en Histoire, Université de Yaoundé I, 2020.

Maladie à coronavirus (COVID 19), 27/03/2020. Disponible sur: www.who.int/fr/emergencies/diseases Consulté le: 13 juin 2020.

La santé mentale et la résilience psychologique pendant la pandémie du COVID 19. 27/03/2020 Disponible sur: www.who.int/fr/healt-topic Consulté le: 13 juin 2020.

BIOS, Chistian. Le Covid 19 et son impact socio-sanitaire au Cameroun: une analyse sociologique d'une tragédie aux conséquences plurielles. Nkafu, s/p., 19/06/2020. Disponible sur: https://nkafu.org/ le-covid-19-et-son-impact-socio-sanitaire-au-cameroun/ Consulté le: 9 juin 2020.

LLYOD, John. Comment l'Union Européenne a abandonné l'Italie. Le Point, Débats, Paris, s./v., s/p, avr. 2020. Disponible sur: https://www.lepoint.fr/debats/comment-l-union-europeenne-a-abandonnel-italie-16-05-2020-2375724_2.php Consulté le: 10 juin 2020.

KHALFALLAH, Amira-Géhanne. COVID 19: L'Afrique l'impossible pronostic. Atalayar, Etre des rives, s./v., s/p, juin 2020. Disponible sur: https://atalayar. $\mathrm{com} / \mathrm{fr} / \mathrm{blog} /$ covid-19-L\%E2\%80\%99afrique-L\%E2\%8 0\%99impossible-pronostic Consulté le: $1^{\text {er }}$ juil. 2020.

CABRIOL, Michel. Comment la France imagine une possible implosion de l'Afrique face au Covid 19. Latribune, Paris, s./v., s/p, abr. 2020. Disponible sur: https://www.latribune.fr/economie/international/ comment-la-france-imagine-une-possible-implosion-de-l-afrique-face-au-covid-19-844055.html Consulté le: 1er juil. 2020.

La COVID-19 va renforcer l'autonomie scientifique de l'Afrique. SciDevNet, mai 2020. Disponible sur: https://www.scidev.net/afrique-sub-saharienne/ editorials/covid-19-renforcer-autonomie-scientifique-pharmaceutique-afrique/ Consulté le: 2 juil. 2020.

Le vaccin d'AstraZeneca pourrait protéger contre le
Covid-19 pendant un an. Juin 2020. Disponible sur: www.challenges.fr/entreprise Consulté le: 11 juil. 2020.

Résilience: définition et application aux domaines de la RSE et du développement durable. Disponible sur: https://youmatter.world/fr/definition/resilience Consulté le 12 juil. 2020.

BELOKO, Fabrice. Coronavirus: le Cameroun s'intéresse à la solution de Monseigneur Kléda. Allo Docteur Africa, s/p., juin 2020. Disponible sur: https:// allodocteurs.africa/actualites/epidemie/covid-19/ coronavirus-le-cameroun-s-interesse-a-la-solution-de-monseigneur-kleda Consulté le 8 août 2020.

NTAP, Emmanuel Jules. Les peuples autochtones camerounais face au coronavirus. Voa Afrique. Cameroon, s./v., s/p, août 2020. Disponible sur: https://www.voaafrique.com/a/r\%C3\%A9siliencedes-peuples-autochtones-au-covid-19-/ 5537652. html 10/08/2020 Consulté le 5 août 2020.

SAVANA, Anna. Covid-19: les 10 pays les plus affectés en Afrique. Financiala Frik. Paris, s./v., s/p, mars 2020. Disponible sur: www.financialafrik.com 23/06/2020 - Consulté le 5 juil. 2020.

Alerte COVID-19. Disponible sur: www.google.com Consulté le 10 juil. 2020.

GOLDSTEINE, Guy-Philippe; ROZEN, Alon. Coronavirus: l'institution ou l'entreprise ne peut se permettre de devenir un nouveau foyer de contagion. Le Monde, Tribune, Paris, s./v., s/p, mars 2020. Disponible sur: www.lemonde.fr Consulté le: 10 juil. 2020.

BARROUX, Rémi. Les mesures de distanciation sociale ne sont pas bien comprises par les Français. Le Monde, Paris, s./v., s/p, mars 2020. Disponible sur: www.lemonde.fr Consulté le 14 août 2020.

RABIYATOU, Mana. Respect de la distanciation physique: le relâchement continue. Cameroon Tribune. Socièté, Cameroon, s./v., s/p, juin 2020. Disponible sur: www.cameroon-tribune.cm/article Consulté le juil. 2020.

NDINGA NGOMA, John. Coronavirus-confinement: la Cameroun se refuse à tout "mimétisme". Afri- 
canews, Paris, s./v., s/p, 26/03/2020. Disponible sur: www.africanews.com Consulté le 8 juil. 2020.

TCHUILEU, Alain. Respect de la distanciation physique: le relâchement continue. Cameroon Tribune. Socièté, Cameroon, s./v., s/p, juilet 2020. Disponible sur: www.cameroon-tribune.cm/article Consulté le: 20 juil. 2020.

Recebido em: 27/07/2020

Revisado em: 08/09/2020

Aprovado em: 18/12/2020

Joseph Yannick Mbatchou est Dr en Histoire Sociale, spécialiste en histoire de la médecine pour le Centre de Recherche de Formation Doctorale en Sciences Humaines, Sociales et Educatives, Faculté des Arts, Lettre et Sciences Humaines, Université de Yaoundé 1. E-mail: joyannmbatchou@yahoo.fr 